\title{
Ebola Infectious Model Based on SEIR
}

\author{
Ji Tao \\ School of North China Electric Power University, Baoding 071000, China \\ 986222908@qq.com
}

Keywords: Ebola; SI model; SEIR model.

\begin{abstract}
This paper build two mathematics models to study the spread of the disease. In model 1, we collect the number of total cases and deaths of Ebola in Guinea, Liberia and Sierra Leone from WHO's website. There is no vaccine or medication which can protect people from the disease in this period, so in order to describe the spread of Ebola virus accurately, we build improved SI epidemic model to estimate how many cases of Ebola will exist in these countries when the epidemic has been controlled (see Table 1). In model 2, we assume the effective medication is widely used to fight against Ebola, and we take patients in the incubation period into consideration. So we build improved SEIR epidemic model. We use the iterative method to obtain the numerical solution for its nonlinearity. We estimate that the number of recovered people is 6071 in Guinea.
\end{abstract}

\section{Introduction}

The 2014 Ebola epidemic is the largest in history, affecting multiple countries in West Africa. The Ebola virus causes an acute, serious illness which is often fatal if untreated. There is no cure for Ebola, nor are there any vaccines that can prevent the disease. So we build improved SI epidemic model to estimate how many cases of Ebola will exist in these countries when the epidemic has been controlled. According to the CDC, research shows that patients who recover from Ebola can develop antibodies that will protect them from the virus for at least 10 years, or possibly even longer. Also, after the patient recovers, he or she is no longer contagious. At present, great progress has been made in the research of Ebola drugs, so we assume the effective medication is widely used to fight against Ebola in model 2 based on SEIR to estimate the number of recovered people

\section{Model of Spread of the Ebola Based on Improved SI}

\subsection{Assumptions.}

We assume those who recover from Ebola will not be infected again and ignore the impact to the spread of the virus because of population flow. In model 1, nobody recovers from the disease before the effective drug was developed. There is no cure for Ebola, nor are there any vaccines that can prevent the disease. Those who recover from the disease do so through the strength of their own immune system and these people is too rare to count.

\subsection{Model of Spread of the Disease Based on Improved SI.}

We collect the number of total cases and deaths of Ebola in Guinea, Liberia and Sierra Leone from WHO's website. In this model, the population of every epidemic area can be divided into the susceptible people and the infectious people. This model labels these two compartments $S=$ number susceptible, I =number infectious. The number infected by a patient is proportional to the number of healthy people per day. We define it as the rate of infection and use $k$ to describe it. Daily death proportion of infectious people

According to the above, we have

$$
\begin{aligned}
& \frac{d s}{d t}=-k S I+\gamma I \\
& \frac{d I}{d t}=k S I-\gamma I \\
& S(\mathrm{t})+\mathrm{I}(\mathrm{t})=\mathrm{N} \\
& \mathrm{I}(0)=\mathrm{I}_{0}>0
\end{aligned}
$$


Solve the equation (1), and we have

$$
I(\mathrm{t})=\frac{I_{0}\left(\mathrm{~N}-\frac{\gamma}{k}\right)}{I_{0}+e^{-(\mathrm{kN}-\gamma) \mathrm{t}}\left(\mathrm{N}-\frac{\gamma}{k}-I_{0}\right)}
$$

And then we have suppose

$$
I(\mathrm{t})=\frac{1}{a e^{-b x}+c}
$$

And we have

$\lim _{t \rightarrow \infty} I(\mathrm{t})=\mathrm{N}-\frac{\gamma}{k}=\frac{1}{c}$

After programming, we can get the solutions as followings:

Table 1 Table of a, b, c

\begin{tabular}{ccccc}
\hline Country & $\mathrm{a}$ & $\mathrm{b}$ & $\mathrm{c}$ & $\lim _{t \rightarrow \infty} I(\mathrm{t})$ \\
\hline Guinea & 0.3985 & 0.02694 & 0.0009246 & 1082 \\
Liberia & 5.744 & 0.04445 & 0.0002021 & 4948 \\
Sierra Leone & 1.187 & 0.0363 & 0.0001299 & 7698 \\
\hline
\end{tabular}

Form the Table 1, we know that $\lim _{t \rightarrow \infty} I(\mathrm{t})$ is the stable value of infectious people if these country cannot get effective treatment all the time. Using the data we collected, we can calculate $\gamma$. And then we obtain $k$ according to the equation (4). In Table 2, we summarize the rate of infection in these countries.

Table 2 Table of $N$, $\gamma$ and $k$

\begin{tabular}{cccc}
\hline Country & $N$ & $\gamma$ & $k$ \\
\hline Guinea & 11474383 & 0.6545387 & $5.7 \times 10^{-8}$ \\
Liberia & 3994000 & 0.54208 & $1.36 \times 10^{-7}$ \\
Sierra Leone & 5743723 & 0.382349 & $6.666 \times 10^{-8}$ \\
\hline
\end{tabular}

The rate of infection $k$ represents hygiene level in the local area. In other words, the greater the value is, the lower the level of local medical and health is. So the value can be also used in the next model. The figure 1 show how I changes with time in Guinea.

This model is simple, typical and applied well to the situation in which there is no cure for Ebola. However, as is mentioned in the background, symptoms of EVD may appear 2 to 21 days after exposure to the Ebola virus. So the model is unreasonable for ignoring the incubation period.

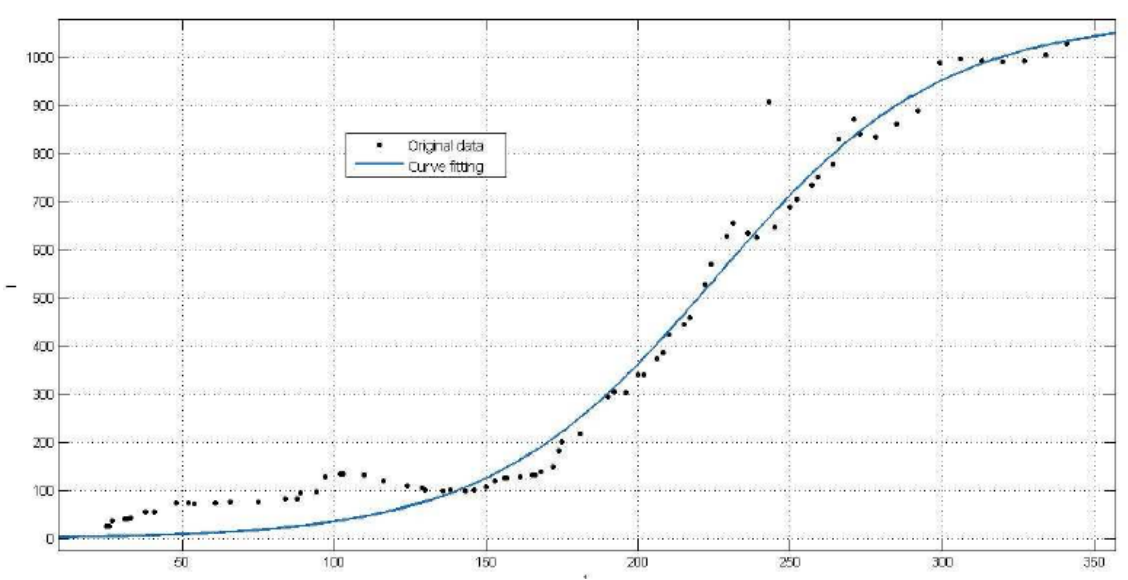

Fig. 1 Fitting figure in Guinea 


\section{Model of Spread of the Ebola Based on SEIR}

In this model, there is adequate medicine transported to the epidemic area and the incubation period is considered. Figure 2 shows the transformation relationship between groups.

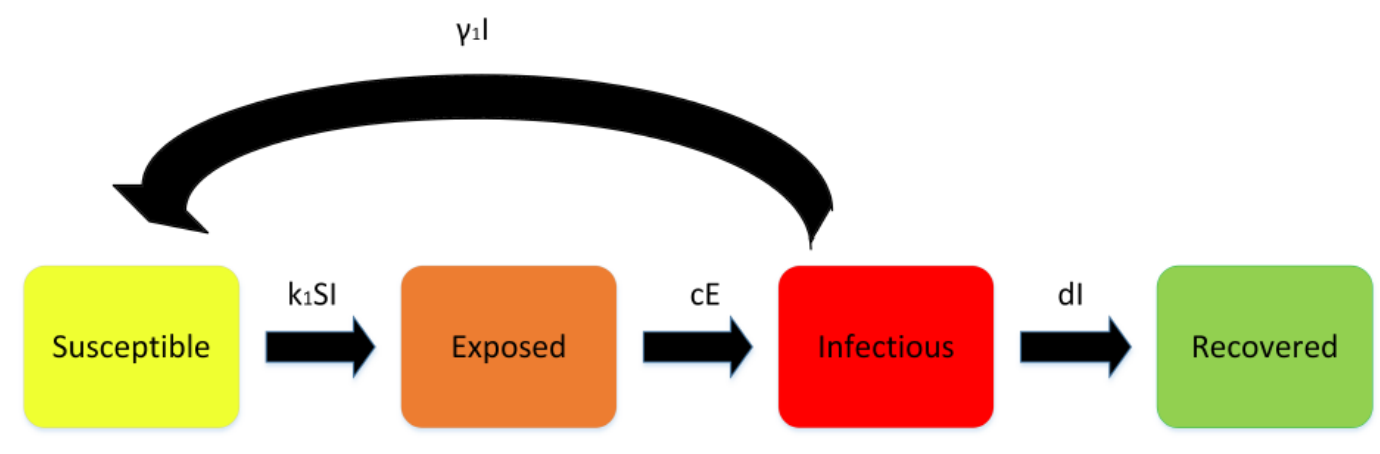

Fig. 2 the transformation relationship between groups

Based on SEIR, We have the equation

$$
\begin{aligned}
& \frac{d S}{d t}=-k_{1} S I+\gamma_{1} I \\
& \frac{d E}{d t}=k_{1} S I-c E \\
& \frac{d I}{d t}=c E-d I+\gamma_{1} I \\
& \frac{d R}{d t}=d I \\
& S(0)=\mathrm{S}_{0}, E(0)=\mathrm{E}_{0}, I(0)=\mathrm{I}_{0}, \mathrm{R}(0)=\mathrm{R}_{0}
\end{aligned}
$$

The number of people who are infected by infectious people and turn into exposed people is $k_{1} S I$, and we have

$$
k_{1}=k e^{-m t}
$$

$k_{1}$ is the rate of infection in this model. $k$ is the value obtained in model $1 . m$ is called consciousness factor which is greater than 0 . Because with the development of the epidemic of diseases and education of the government and the WHO organization, people's awareness of illness is more and more deep and they will avoid infection deliberately.

We deem that the number of people dying of Ebola is $\gamma_{1} I$, and we have

$\gamma_{1}=\gamma e^{-n t}$

$\gamma_{1}$ is the rate of infectious people dying of Ebola per day in this model. $\gamma$ is the value obtained in model 1. $\gamma_{1}$ will be more and more small because of the new medication from the world medical association.

$c$ is daily morbidity proportion, we can stipulate that daily morbidity proportion is a random value between 0.25 to 0.75 . So we have

$c=0.5 \times$ random $(0,1)+0.25$

$d$ is the cure rate of infectious people.

$d=1-\left(1-\mathrm{d}_{0}\right) \mathrm{e}^{-p t}$

We use the iterative method and take Guinea as an example to solve the model. Finally, we can obtain the following results: It takes 25 days to eradicate Ebola since the new medication was delivered to the epidemic area. The number of recovered people $\mathrm{R}$ is 6071 . 


\section{Summary}

We build SI and SEIR models to study the spread of Ebola and calculate the number of infections and recovered people by using iterative method. The models can be referred when studying other infectious diseases

\section{References}

[1]. Deng Mengwei. Several kinds of infectious disease model and research [D]. Northeastern university: northeastern university press, 2006.

[2]. Information on: https://www.cia.gov/library/publications/the-world-factbook/geos/gv.html

[3]. Information on: http://apps.who.int/ebola/en/current-situation/ebola-situation-report 